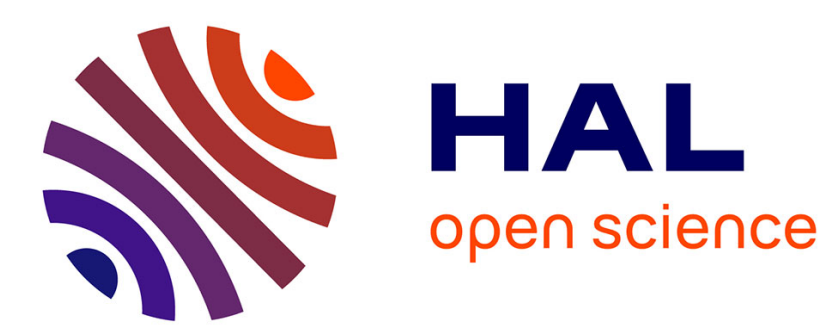

\title{
Error estimation and time-space parameters optimization for FEM non-linear computation
}

L. Gallimard, Pierre Ladevèze, Jean-Pierre Pelle

\section{To cite this version:}

L. Gallimard, Pierre Ladevèze, Jean-Pierre Pelle. Error estimation and time-space parameters optimization for FEM non-linear computation. Computers \& Structures, 1997, 64 (1-4), pp.145-156. hal-02003789

\section{HAL Id: hal-02003789 \\ https://hal.science/hal-02003789}

Submitted on 1 Feb 2019

HAL is a multi-disciplinary open access archive for the deposit and dissemination of scientific research documents, whether they are published or not. The documents may come from teaching and research institutions in France or abroad, or from public or private research centers.
L'archive ouverte pluridisciplinaire HAL, est destinée au dépôt et à la diffusion de documents scientifiques de niveau recherche, publiés ou non, émanant des établissements d'enseignement et de recherche français ou étrangers, des laboratoires publics ou privés. 


\title{
ERROR ESTIMATION AND TIME-SPACE PARAMETERS OPTIMIZATION FOR FEM NON-LINEAR COMPUTATION
}

\author{
L. Gallimard, P. Ladevèze and J. P. Pelle \\ Laboratoire de Mécanique et Technologie, ENS de Cachan/CNRS/Université P. et M. Curie, \\ 61 avenue du Président Wilson, 94235 Cachan Cedex, France
}

\begin{abstract}
In this paper, two adaptive methods which permit the control of the parameters of a finite element computation for time-dependent material models are proposed. These methods use a global error measure in the constitutive relation based on Drucker's inequality. This error includes the accuracy of both the finite element model and the algorithm being used, over the studied time interval. In order to master the mesh element size and the time increments length, an error estimator, which permits the estimation of the errors due to the time discretization, is proposed. Various examples show the reliability of these procedures.
\end{abstract}

\section{INTRODUCTION}

Today, the construction and numerical simulation of models is, more than ever, a major activity in the field of mechanics. A constant concern in both industrial and research environments has been the control of these models, which nowadays can reach very high levels of complexity. A new factor, introduced over the past 15 years, has been the development of truly quantitative tools for testing the quality of FE models (see e.g. Refs [1-3]). This has made it possible to optimize the FE parameters for a prescribed quality level. Of course, the reference remains the original "continuum mechanics" model.

One important research topic is the mastering of finite element computations for non-linear analyses. Few studies have dealt with this question in spite of its practical importance [4-14]. The a posteriori error estimate method developed at Cachan, which is based on the concept of error on constitutive relation and on explicit techniques for the construction of admissible stress and displacement fields, offers a suitable framework for the study of non-linear and time-dependent mechanical models.

The purpose of the present study is to show how it is possible to extend this error estimate method to most cases of non-linearity encountered in structural mechanics for small displacement problems and for quasi-static loading. We focus on the construction of basic tools, namely the error or error indicators, which are capable of evaluating the accuracy of both the FE model (parameters: $h, p$ ) and the numerical solution process being used (parameters: increments, iterations) over time interval $[0, T]$. An important part of the work concerns the application of these tools to optimize calculation parameters, that is, to control the time-space parameters (mesh and increment length). In elasto-plasticity, the quality of the finite element solution at the instant $t$ does not only depend on the quality of the mesh, but also on the quality of the time discretization used from the beginning of the loading. To master a non-linear computation, it is necessary to build error measures that allow taking all the errors of discretization into account over the entire time interval $[0, T]$ : errors due to the mesh, errors due to the incremental method (including the errors introduced by the use of Newton's method on each time increment). Related to the study [15], we propose new examples to show the possibilities of our approach. These examples involve complex loadings as well as significant plastic zones.

The error $\epsilon$ used in this work has been proposed by Ladevèze [16]. This error measure in constitutive relation is based on Drucker's inequality [17], which is satisfied by most of the elasto-plastic and visco-plastic material models. This error simultaneously takes into account the errors due to the space discretization and the errors due to the incremental method.

In order to control the computation parameters, it is necessary to separate the contribution due to the incremental method from the contribution due to the mesh. Hence techniques based on the concept of error in constitutive relation have been elaborated in order to control the quality of the time discretization. We use these techniques to optimize the time discretization and the space discretization separately, as well as to optimize all the computation parameters simultaneously. 


\section{NOTATION}

For the sake of simplicity, let us consider the problem of the analysis of a structure in two-dimensional elasto-plasticity (plane stress or plane strain). Let $[0, T]$ be the time interval. Let us suppose that the structure is a domain $\Omega$. On a part $\partial_{1} \Omega$ of the boundary $\partial \Omega$, we suppose that the imposed displacement field is $U(t, M)=U_{\mathrm{d}}(t, M)$. On the complementary part $\partial_{2} \Omega$, a density of forces $F_{\mathrm{d}}(t, M)$ is imposed. Moreover, $\boldsymbol{\Omega}$ is subjected to a density of body forces $f_{\mathrm{d}}(t, M)$.

The elasto-plastic problem can be formulated in the following manner. Find a displacement field $U$ and a stress field $\sigma$ defined on $[0, T] \times \Omega$ such that:

(1) $U$ satisfies the kinematic constraints

$$
U(t, M)=U_{\mathrm{d}}(t, M) \quad \text { on } \quad \partial_{1} \Omega \quad \forall t \in[0, T],
$$

(2) $\sigma$ satisfies the equilibrium equations

$$
\int_{\Omega} \sigma^{\mathrm{T}} \epsilon\left(U^{*}\right) \mathrm{d} \Omega=\int_{\Omega} f_{\mathrm{d}}^{\mathrm{T}} U^{*} \mathrm{~d} \Omega+\int_{i_{i_{2} \Omega}} F_{\mathrm{d}}^{\mathrm{T}} U^{*} \mathrm{~d} S
$$

$\forall U^{*}$ such that $U^{*}=0$ on $\partial_{1} \Omega \quad \forall t \in[0, T]$,

(3) $\sigma$ and the strain $\epsilon(U)$ satisfy the constitutive relation

$$
\sigma(t, M)=\mathbf{A}\left[\epsilon(U)\left(t^{\prime}, M\right), t^{\prime} \leqslant t\right] \text { in } \Omega \quad \forall t \in[0, T]
$$

where $\mathbf{A}$ is an operator characteristic of the material. The problem eqn (1), is the reference problem and it is solved in an approximate manner by using the incremental method and a finite element discretization.

Assuming that the history of the displacements and of the stresses is known until $t_{i}$, the problem is then to compute this history on the increment $\left[t_{i}, t_{i+1}\right]$. Numerous algorithms, presented in the bibliography, permit the solution of this problem [18, 19].

At the end of each time increment $t_{i+1}$, these algorithms give:

- a finite element displacement field that satisfies the kinematic constraints

$$
U_{\mathrm{h}}\left(t_{i+1}, M\right)=\mathbf{N}(M) \mathbf{q}\left(t_{i+1}\right),
$$

where $\mathbf{N}(M)$ denotes the matrix of the shape functions and $q\left(t_{i+1}\right)$ the vector of the nodal displacements at $t_{i+1}$;

- a stress field $\sigma_{\mathrm{h}}\left(t_{i+1}, M\right)$ which satisfies the equilibrium equation for the finite element problem at $t_{i+1}$ :

$$
\begin{gathered}
\int_{\Omega} \sigma_{\mathrm{h}}\left(t_{i+1}, M\right)^{\mathrm{T}} \mathbf{B}(M) \mathbf{q}^{*} \mathrm{~d} \Omega \\
=\int_{\Omega} f_{\mathrm{d}}\left(t_{i+1}, M\right)^{\mathrm{T}} \mathbf{N}(M) \mathbf{q}^{*} \mathrm{~d} \Omega \\
\quad+\int_{\tau_{2} \Omega} F_{\mathrm{d}}\left(t_{i+1}, M\right)^{\mathrm{T}} \mathbf{N}(M) \mathbf{q}^{*} \mathrm{~d} S \\
\forall U_{\mathrm{h}}^{*}=\mathbf{N}(M) \mathbf{q}^{*} \text { such that } \\
U_{\mathrm{n}}^{*}=0 \text { on } \partial_{1} \Omega,
\end{gathered}
$$

where $\mathbf{B}(M) \mathbf{q}^{*}$ is the strain associated with the field $U_{\mathrm{h}}^{*}=\mathbf{N}(M) \mathbf{q}^{*}$.

Remark: the stress field given by the finite element solver is very often the field $\tilde{\sigma}_{\mathrm{h}}\left(t_{i+1}, M\right)$ obtained from $\epsilon\left(U_{\mathrm{h}}\right)$ by integrating the constitutive relation

$$
\tilde{\sigma}_{\mathrm{h}}\left(t_{i+1}, M\right)=\mathbf{A}\left(\epsilon\left(U_{\mathrm{h}}\right)(\tau, M), \tau \leqslant t_{i+1}\right) .
$$

It must be noted that this stress field satisfies eqn (3) in an approximate manner.

\section{ERROR IN CONSTITUTIVE RELATION FOR LINEAK COMPUTATIONS}

Let us simplify the problem eqn (1) by taking $\mathbf{A}$ as a linear constitutive relation:

$$
\sigma=K_{\mathrm{e}} \epsilon,
$$

where $K_{\mathrm{e}}$ is the Hooke's tensor in elasticity.

Let $\left(U_{\mathrm{KA}}, \sigma_{\mathrm{SA}}\right)$ be a displacement-stress pair, where $U_{\mathrm{KA}}$ is a kinematically admissible displacement field eqn (1a) and $\sigma_{\mathrm{SA}}$ satisfies the equilibrium eqn (1b). Generally, this pair does not satisfy the constitutive relation. The pair $\left(U_{\mathrm{KA}}, \sigma_{\mathrm{SA}}\right)$ is the exact solution of the problem, if and only if it satisfies the constitutive relation

$$
\sigma_{\mathrm{SA}}-K_{\mathrm{e}} \epsilon\left(U_{\mathrm{KA}}\right)=0 .
$$

If that equality is not satisfied, then the pair is only an approximate solution of the problem and the quantity

$$
\sigma_{\mathrm{SA}}-K_{e} \epsilon\left(U_{\mathrm{KA}}\right),
$$

which is referred to as the error in constitutive relation [1], chosen to evaluate the quality of the pair $\left(U_{\mathrm{KA}}, \sigma_{\mathrm{SA}}\right)$. In elasticity, we use the energy norm over the whole structure to evaluate this error:

$$
\mathbf{e}=\left\|\sigma_{\mathrm{SA}}-K_{\epsilon} \epsilon\left(U_{\mathrm{KA}}\right)\right\|_{\mathbf{\Omega}},
$$


where

$$
\|\sigma\|_{\Omega}^{2}=\int_{\Omega} \sigma^{\top} K_{e}^{-1} \sigma \mathrm{d} \Omega
$$

Associated with this global error, a relative error is defined:

$$
\epsilon=\frac{\mathbf{e}}{\left\|\sigma_{\mathrm{SA}}+K_{\mathrm{e}} \epsilon\left(U_{\mathrm{KA}}\right)\right\|_{\mathbf{\Omega}}},
$$

and the contribution to the global error of an element $E$ of $\Omega$

$$
\epsilon_{E}=\frac{\left\|\sigma_{\mathrm{SA}}-K_{\mathrm{e}} \epsilon\left(U_{\mathrm{KA}}\right)\right\|_{E}}{\left\|\sigma_{\mathrm{SA}}+K_{\mathrm{e}} \epsilon\left(U_{\mathrm{KA}}\right)\right\|_{\Omega}}
$$

Then we obtain

$$
\epsilon^{2}=\sum_{E} \epsilon_{E}^{2}
$$

The contribution of each element of the mesh to the global error is a local measure of the solution quality.

\section{ERROR IN CONSTITUTIVE RELATION FOR NON-LINEAR COMPUTATIONS}

\subsection{Global error}

Let $\left(U_{\mathrm{KA}}, \sigma_{\mathrm{SA}}\right)$ be a displacement-stress pair, which is zero at $t=0$. $U_{\mathrm{KA}}$ is a kinematically admissible displacement field eqn (1a), and $\sigma_{\mathrm{SA}}$ satisfies the equilibrium eqn (1b). Generally, this pair does not satisfy the constitutive relation (1c). Thus $\left(U_{\mathrm{KA}}, \sigma_{\mathrm{SA}}\right)$ is an approximate solution of the problem eqn (1).

The strain field $\epsilon_{\mathrm{KA}}=\epsilon\left(U_{\mathrm{KA}}\right)$ can be related to a stress field $\sigma_{\mathrm{KA}}$ through the constitutive relation. In the same way, the stress $\sigma_{\mathrm{SA}}$ can be related to a strain field $\epsilon_{S A}$ through the inverse of the constitutive relation.

We define the quantity $\eta(t, M)$ as follows:

$$
\eta(t, M)=\int_{0}^{t}\left(\sigma_{\mathrm{SA}}-\sigma_{\mathrm{KA}}\right)^{\mathrm{T}}\left(\dot{\epsilon}_{\mathrm{SA}}-\dot{\epsilon}_{\mathrm{KA}}\right) \mathrm{d} \tau
$$

For a material which strictly satisfies Drucker's inequality $[17,20], \eta(t, M)$ is greater than or equal to zero, and the pair $\left(U_{\mathrm{KA}}, \sigma_{\mathrm{SA}}\right)$ is the exact solution of the problem eqn (1), if and only if

$$
\eta(t, M)=0 \quad \forall t \in[0, T] \text { and } \forall M \in \Omega
$$

Most constitutive laws used in plasticity and in visco-plasticity strictly satisfy Drucker's inequality, as do, for instance, Prandtl-Reuss's model which will be used for the examples.
To estimate the quality of $\left(U_{\mathrm{KA}}, \sigma_{\mathrm{SA}}\right)$ as an approximate solution to the problem eqn (1), the previous relations lcad us to define the following error measure:

$$
\mathbf{e}=\left[\operatorname{Sup}_{i \in[0, I]} \int_{\Omega} \eta(t, M) \mathrm{d} \Omega\right]^{1 / 2}
$$

so that

$$
\mathbf{e}=0 \Leftrightarrow\left(U_{\mathrm{KA}}, \sigma_{\mathrm{SA}}\right)
$$

is the exact solution of problem eqn (1).

e is called the error in constitutive relation associated with the admissible pair $\left(U_{\mathrm{KA}}, \sigma_{\mathrm{SA}}\right)$

Associated with this absolute global error, a relative global error can be defined:

$$
\epsilon=\frac{\mathrm{e}}{D}
$$

where

$$
D=\left[2 \operatorname{Sup}_{\ell \in[0, T]} \int_{\Omega} \int_{0}^{t}\left(\sigma_{\mathrm{SA}}^{\mathrm{T}} \dot{\epsilon}_{\mathrm{SA}}+\sigma_{\mathrm{KA}}^{\mathrm{T}} \dot{\epsilon}_{\mathrm{KA}}\right) \mathrm{d} \tau \mathrm{d} \Omega\right]^{1 / 2}
$$

Remark: if we consider a monotonous loading and suppose that the whole structure remains elastic, then it can be shown [15] that the error in the constitutive relation defined in eqn (13) is equal to the energy errors classically used, see eqn $(7)[4,21]$.

\subsection{Time error indicator}

The error measure in the constitutive relation $\epsilon$ is global in space and in time. It simultaneously takes into account the errors due to the space discretization and the errors due to the incremental method. To develop efficient adaptivity techniques for non-linear problems, it is generally insufficient to control only the size of the mesh elements; it is also necessary to control the size of the time steps. To achieve this goal, it is essential to be able to separate, in the global error $\epsilon$, the part of the error due to the spatial discretization from the part of the error due to the time discretization. We propose herein a very simple time error indicator which allows us to estimate the part of the error due to the time discretization.

Let us consider the pair $\left(U_{\mathrm{KA}}, \sigma_{\mathrm{h}}\right)$ where $U_{\mathrm{KA}}$ is a kinematically admissible field that satisfies eqn (1a), and where $\sigma_{\mathrm{h}}$ is the stress field defined by

$$
\begin{aligned}
& \sigma_{\mathrm{h}}(t, M)=\sigma_{\mathrm{h}}\left(t_{i}, M\right)+\frac{t-t_{i}}{t_{i+1}-t_{i}} \\
& \quad \times\left[\sigma_{\mathrm{h}}\left(t_{i+1}, M\right)-\sigma_{\mathrm{h}}\left(t_{i}, M\right)\right] \quad \forall t \in\left[t_{i}, t_{i+1}\right],
\end{aligned}
$$


where the fields $\sigma_{\mathrm{h}}\left(t_{i+1}, M\right)$ satisfy the equilibrium equation of the finite element model, eqn (3), at the end of each increment.

Let us consider the problem eqn (16) obtained from the reference problem eqn (1) by a finite element spatial discretization. Find $U_{\mathrm{h}}(t, M)=\mathbf{N}(M) \mathbf{q}(t)$ and $\sigma_{\mathrm{h}}(t, M)$ such that:

(I) $U_{\mathrm{h}}$ satisfies the kinematic constraints

$$
U_{\mathrm{n}}(t, M)=U_{\mathrm{d}}(t, M) \quad \text { on } \quad \partial_{1} \Omega \quad \forall t \in[0, T]
$$

(2) $\sigma_{\mathrm{h}}$ satisfies the equilibrium equations of the finite element model

$$
\begin{aligned}
& \int_{\Omega} \sigma_{\mathrm{h}}(t, M)^{\mathrm{T}} \mathbf{B}(M) \mathbf{q}^{*} \mathrm{~d} \Omega \\
& =\int_{\Omega} f_{\mathrm{d}}(t, M)^{\mathrm{T}} \mathbf{N}(M) \mathbf{q}^{*} \mathrm{~d} \Omega \\
& \quad+\int_{i_{2} \Omega} F_{\mathrm{d}}(t, M)^{\mathrm{T}} \mathbf{N}(M) \mathbf{q}^{*} \mathrm{~d} S \\
& \forall t \in[0, T] \quad \forall U_{\mathrm{h}}^{*}(M)=\mathbf{N}(M) \mathbf{q}^{*} \\
& \text { such that } U_{\mathrm{h}}^{*}=0 \text { on } \partial_{1} \Omega,
\end{aligned}
$$

(3) $\sigma_{\mathrm{h}}$ and the strain $\epsilon\left(U_{\mathrm{h}}\right)$ satisfy the constitutive relation

$$
\sigma_{\mathrm{h}}(t, M)=\mathbf{A}\left[\epsilon\left(U_{\mathrm{h}}\right)\left(t^{\prime}, M\right), t^{\prime} \leqslant t\right] \text { in } \Omega
$$

$$
\forall t \in[0, T] .
$$

The pair $\left(U_{\mathrm{KA}}, \sigma_{\mathrm{h}}\right)$ thus constructed satisfies all of problem eqns (16), except the constitutive relation (16c). The quality of this pair as an approximate solution to the problem eqn (16) may be estimated by an error measure built on Drucker's inequality.

Let $\epsilon_{\mathrm{h}}$ be a strain field computed from $\sigma_{\mathrm{h}}$ by an integration of the constitutive law. Then the pairs $\left(\epsilon_{\mathrm{h}}, \sigma_{\mathrm{h}}\right)$ and $\left[\epsilon\left(U_{\mathrm{KA}}\right), \sigma_{\mathrm{KA}}\right]$ satisfy the constitutive law. The associated error in constitutive law is defined by

$$
\mathbf{i}_{\mathrm{time}}=\left[\operatorname{Sup}_{t \in[0, \Pi} \int_{\Omega} \bar{\eta}(t, M) \mathrm{d} \Omega\right]^{1 / 2},
$$

where

$$
\bar{\eta}(t, M)=\int_{0}^{1}\left(\sigma_{\mathrm{h}}-\sigma_{\mathrm{KA}}\right)^{\mathrm{T}}\left(\dot{\epsilon}_{\mathrm{h}}-\dot{\epsilon}\left(U_{\mathrm{KA}}\right)\right) \mathrm{d} \tau
$$

For a material which strictly satisfies Drucker's inequality, $i_{t m e}=0$ if and only if the pairs $\left(c_{h}, \sigma_{h}\right)$ and
$\left(\epsilon\left(U_{\mathrm{KA}}\right), \sigma_{\mathrm{KA}}\right)$ are equal, that is if and only if the pair $\left(U_{\mathrm{KA}}, \sigma_{\mathrm{h}}\right)$ is the exact solution to the problem eqn (16).

Hence, for a given spatial discretization, $\mathbf{i}_{\text {sime }}$ estimates the errors due to the time discretization: the incremental method and Newton's algorithm. The absolute error $\mathbf{i}_{\text {time }}$ can be associated, as for $\mathbf{e}$, to a relative error:

$$
i_{\text {time }}=\frac{\mathbf{i}_{\text {time }}}{D_{\text {time }}},
$$

with

$$
D_{\text {time }}=\left[2 \operatorname{Sup}_{i \in[0, I]} \int_{\Omega} \int_{0}^{t}\left(\sigma_{\mathrm{KA}}^{\mathrm{T}} \epsilon_{\mathrm{KA}}+\sigma_{\mathrm{h}}^{\mathrm{T}} \dot{\epsilon}\left(U_{\mathrm{h}}\right)\right) \mathrm{d} \tau \mathrm{d} \Omega\right]^{1 / 2} .
$$

Hence $i_{\text {time }}$ estimates the quality of the approximate solution computed as an approximate solution to the problem eqn (16). In practice, we will use $\mathbf{i}_{\text {time }}$ or the relative quantity $i_{\text {time }}$ as an error indicator to evaluate the part of the error due to the time discretization.

\subsection{Application to finite element computation}

The displacement fields $U_{\mathrm{h}}\left(t_{i}, M\right)$ obtained by a finite element computation satisfy the kinematic constraints. Under the assumption, which is not very restrictive in practice, that the displacement field $U_{\mathrm{d}}(t, M)$ given on $\partial_{1} \Omega$ is linear on each time increment $\left[t_{i}, t_{i+1}\right]$, the field $U_{\mathrm{KA}}$ can be chosen for $t \in\left[t_{i}, t_{i+1}\right]$ equal to

$$
\begin{aligned}
& U_{\mathrm{KA}}(t, M)=U_{\mathrm{h}}\left(t_{i}, M\right) \\
& \quad+\frac{t-t_{i}}{t_{i+1}-t_{i}}\left(U_{\mathrm{h}}\left(t_{i+1}, M\right)-U_{\mathrm{h}}\left(t_{i}, M\right)\right) .
\end{aligned}
$$

On the other hand, the calculated stress $\sigma_{\mathrm{h}}$ is not statically admissible. So, it is necessary to build, with a post-processor of the finite element analysis, a stress field $\sigma_{\mathrm{SA}}$ that satisfies the equilibrium equations over the interval $[0, T]$.

Over the past several years we have developed techniques for constructing admissible fields for the evaluation of the errors in linear analysis. These techniques are completely independent of the constitutive relation. They will be used herein to build a stress field $\sigma_{\mathrm{SA}}\left(t_{i+1}\right)$ at the end of each time step $t_{i+1}$ that satisfies the equilibrium eqn (1b) $[4,21]$. The main lines of the construction are summarized in the Scetion 4.4. Then, if we define for $t \in\left[t_{i}, t_{i+1}\right]$

$$
\begin{aligned}
& \sigma_{\mathrm{SA}}(t, M)=\sigma_{\mathrm{SA}}\left(t_{i}, M\right) \\
& +\frac{t-t_{i}}{t_{i+1}-t_{i}}\left[\sigma_{\mathrm{SA}}\left(t_{i+1}, M\right)-\sigma_{\mathrm{SA}}\left(t_{i}, M\right)\right],
\end{aligned}
$$


and under the assumption that the loading is linear on each time increment (an assumption that is not very restrictive in practice) we obtain a field $\sigma_{\mathrm{SA}}$ which satisfies the equilibrium eqn (lb) at each moment.

Remark: the procedure of constructing $\sigma_{\mathrm{SA}}\left(t_{t+1}, M\right)$ uses the equilibrium equation of the finite element model, eqn (3), which is verified by $\sigma_{\mathrm{h}}\left(t_{i+1}, M\right)$. If the finite element software gives the field $\tilde{\sigma}_{h}\left(t_{i+1}, M\right)$ defined by eqn (4) which satisfies eqn (3) only approximately, it is necessary to build a field $\sigma_{\mathrm{h}}\left(t_{i+1}, M\right)$ which satisfies exactly eqn (3). Such a field can easily be computed in a post-processor by partially completing an additional iteration of Newton's method.

\subsection{Construction of an admissible field}

We would like to highlight herein the main aspects of the construction of an admissible stress field. For a non-linear model, this construction occurs at each instant $t_{i}$ of the time discretization.

The problem is to find a stress field $\sigma_{\mathrm{SA}}\left(t_{i}\right)$ such that

$$
\begin{gathered}
\forall U^{*} \text { such that } U^{*}=0 \text { on } \partial_{1} \Omega \\
\int_{\Omega} \sigma_{\mathrm{SA}}\left(t_{i}, M\right)^{\mathrm{T}} \epsilon\left(L^{*}\right) \mathrm{d} \Omega=\int_{\Omega} f_{\mathrm{d}}\left(t_{i}, M\right)^{\mathrm{T}} U^{*} \mathrm{~d} \Omega \\
+\int_{\partial_{2} \Omega} F_{\mathrm{d}}\left(t_{i}, M\right)^{\mathrm{T}} U^{*} \mathrm{~d} S .
\end{gathered}
$$

Hence

$$
\begin{cases}\operatorname{div} \sigma_{\mathrm{SA}}\left(t_{i}, M\right)+f_{\mathrm{d}}\left(t_{i}, M\right)=0 & \text { on } \Omega \\ \sigma_{\mathrm{SA}}\left(t_{i}, M\right) \mathrm{n}=F_{\mathrm{d}}\left(t_{i}, M\right) & \text { on } \partial_{2} \Omega\end{cases}
$$

where $\mathbf{n}$ denotes the unitary normal outside vector.

Moreover, we impose $\sigma_{\mathrm{SA}}\left(t_{i}\right)$ to be linked to $\sigma_{\mathrm{h}}\left(t_{i}\right)$ by the following condition:

$$
\int_{E}\left[\sigma_{\mathrm{SA}}\left(t_{i}, M\right)-\sigma_{\mathrm{h}}\left(t_{i}, M\right)\right]^{\mathrm{T}} \epsilon\left(\phi_{j}\right) \mathrm{d} E=0,
$$

which must be satisfied for all functions of basis $\phi_{j}$ associated with the finite element discretization.

In an initial step, we determine on the edges of each element the force distributions $\eta_{E} \hat{F}\left(t_{i}, M\right)$ that equilibrate the body forces $f_{\mathrm{d}}\left(t_{i}, M\right) . \eta_{E}= \pm 1$ and is constant on each edge of $E$. Moreover, on the edge common to two elements $E$ and $E^{\prime}$, we have $\eta_{E}+\eta_{E^{*}}=0$.

In a following step, the stress field $\sigma_{\mathrm{SA}}\left(t_{i}, M\right)$ is built on each element $E$ from the force distributions
$\eta_{E} \hat{F}\left(t_{i}, M\right)$, by determining a simple solution to the equilibrium equations:

$$
\begin{cases}\operatorname{div} \sigma_{\mathrm{SA}}\left(t_{i}, M\right)+f_{\mathrm{d}}\left(t_{i}, M\right)=0 & \text { in } E, \\ \sigma_{\mathrm{SA}}\left(t_{i}, M\right) \mathbf{n}=\eta_{E} \hat{F}\left(t_{i}, M\right) & \text { on } \partial E .\end{cases}
$$

\section{ADAPTIVITY OF COMPUTATIONS}

In order to control the parameters of an elasto-plastic computation, it is necessary to separate in the global error the part due to the time discretization from the part due to the space discretization.

\subsection{Evolutions of the errors as a function of $\mathrm{h}$ and $\Delta \mathrm{t}$}

The error e takes into account the errors due to both the mesh and the time discretization. To separate these contributions, we suppose that the global error e may be split into two parts:

$$
\mathbf{e}^{2}=\mathbf{I}_{\text {space }}^{2}+\mathbf{I}_{\text {time }}^{2}
$$

where $I_{\text {space }}$ denotes the contribution of the errors due to the spatial discretization and $\mathbf{I}_{\text {time }}$ the contribution of the errors of discretization over time.

Based on numerical experiments [15], we have assumed that the time indicator $\mathbf{i}_{\text {time }}$, eqn (17), is a good estimate of $\mathbf{I}_{\text {time }}$. Thus, the part of the error due to space may be defined by

$$
\mathbf{I}_{\text {space }}^{2}=\operatorname{Sup}\left\{\mathbf{e}^{2}-\mathbf{i}_{\text {time }}^{2}, 0\right\}
$$

Remark: for each example studied, we have always noted the inequality $\mathbf{e}^{2}>\mathbf{i}_{\text {time. }}^{2}$. If this inequality holds, we have $I_{\text {space }}^{2}=\mathrm{e}^{2}-\mathbf{i}_{\text {time. }}^{2}$. Nevertheless, as the inequality $\mathbf{e}^{2}>\mathbf{i}_{\text {time }}^{2}$ has not been proved, the definition from eqn (27) insures that $\mathbf{I}_{\text {space }}^{2}$ is greater than or equal to zero.

To predict a new mesh from $\mathbf{I}_{\text {space }}$ and a new time discretization from $\mathbf{I}_{\text {time }}$, we must know the behaviour of these errors as functions of both the element size $h$ and the time increment size $\Delta t$. As far as we know, theoretical results on this subject do not exist, so we have estimated these types of behaviour with numerical tests.

To evaluate the behaviour of $\mathbf{I}_{\text {time }}$, we need in fact to evaluate the behaviour of $i_{\text {time }}$ as a function of the time step size $\Delta t$. The numerical tests that have been conducted show that

$$
i_{\text {time }}=O(\Delta t)
$$

As an example, let us consider the frame beam shown in Fig. 1a. The mesh used (447 three-node triangular elements) is given in Fig. $1 \mathrm{~b}$, and the loading is monotonous. The analysis is conducted for various values of the number of time steps. The evolution of $i_{\text {time }}$ as a function of the number of time steps is shown 
in Fig. 1c, and the size of the plastic zone is given in Fig. 1d. It can be noticed that the evolution of $i_{\text {time }}$ as a function of the number of time steps is consistent with the condition from eqn (28).

Numerical tests [15] have shown that it is reasonable to consider that the part of the error due to the spatial discretization $I_{\text {space }}$ evolves, relatively to the size of the elements, much in the same way as the error in elasticity:

$$
\mathbf{I}_{\text {space }}=O\left(h^{q}\right)
$$

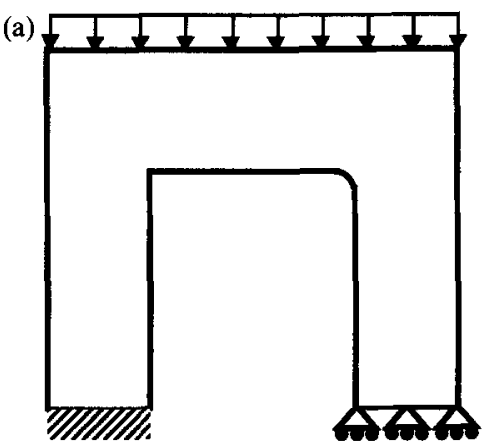

(b)

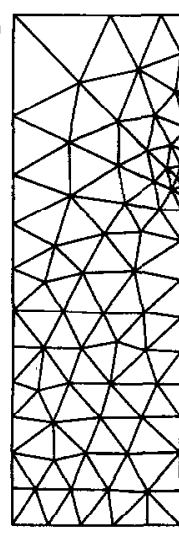

(c)

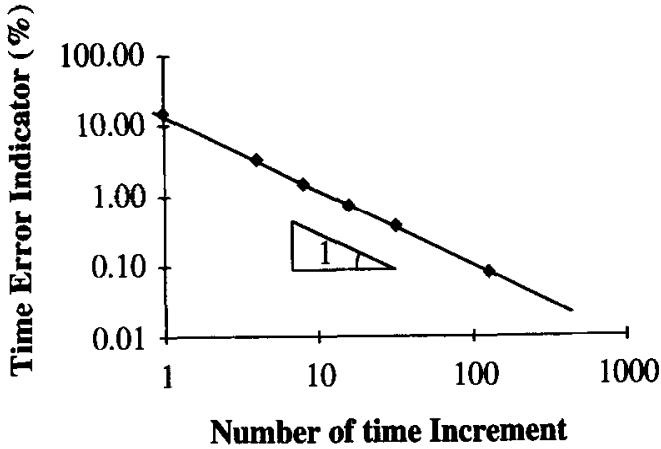

(d)
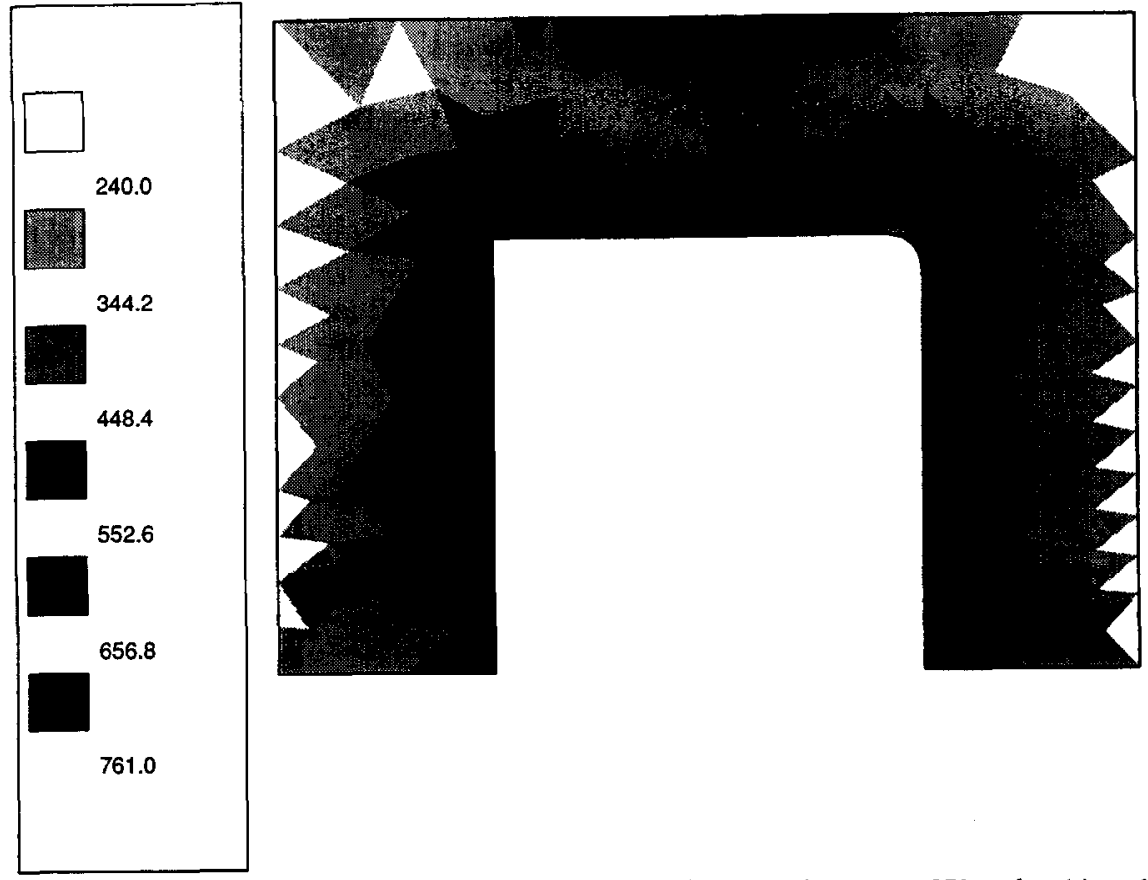

Fig. 1. (a) Mechanical problem; (b) mesh -447 three-node triangular elements - 270 nodes; (c) evolution of $i_{\text {time; }}$ (d) size of the plastic zone-initial threshold $240 \mathrm{MPa}$. 
where $q$ depends on the type of elements used, but equally on the regularity of the solution.

\subsection{Adaptation of the space parameters}

The first proce:dure consists of using sufficiently fine time discretization in order to neglect the part of the error due to time as compared with the error due to the space discretization. This procedure may be described in the following way. Let $\epsilon_{0}$ be the prescribed accuracy:

(1) An initial analysis over the time interval $[0, T]$ is performed on a mesh $T_{e}$ (previously optimized in elasticity) for a time discretization $\Delta$. Then the global error $\epsilon$ and the indicator in time $i_{\text {time }}$ are computed.

(2) If $i_{\text {time }} \leqslant \theta \epsilon_{0}$, then it is considered that the errors due to the time discretization may be neglected. The characteristics of the optimal mesh $\mathrm{T}^{*}$ are determined by using the procedure developed in elasticity $[4,21]$.

(3) If $i_{\text {time }}>\theta \epsilon_{0}$, then a new time discretization $\Delta^{*}$ is determined in the part of $[0, T]$ where the loading leads to a plastic behaviour, with the aim of satisfying $i_{\text {tme }}^{*} \leqslant \theta \epsilon_{0}$. The size $\Delta t^{*}$ of the time steps is computed by using eqn (28):

$$
\Delta t^{*}=\frac{\theta \epsilon_{0}}{i_{\text {time }}} \Delta t
$$

where $\Delta t$ is the size of the time steps during the initial computation.

A new analysis is performed with $\Delta^{*}$ on the initial mesh $\mathbf{T}_{\mathbf{e}}$. In one step, this procedure generally leads to the obtention of the desired inequality and step (2) above is then applied. In this procedure, $\theta$ is a parameter. The different examples computed have led us to choose $\theta$ approximately equal to $1 / 3$. To illustrate this procedure, we have chosen two examples.

Firstly, let us consider the mechanical problem shown in Fig. 2a. The loading is non-monotonous and is shown in Fig. 2b. The initial mesh used (Fig. 2c) has 278 six-node triangular elements. The prescribed accuracy is $\epsilon_{0}=5 \%$. The errors computed are (Fig. 2d) $\epsilon==11.4 \%$ and $i_{\text {time }}=0.53 \%$. In this initial analysis, $i_{\text {tine }}$ can be neglected, as $i_{\text {time }} \leqslant \frac{1}{3} \epsilon_{0}$, and step (2) described above may be applied. The optimized mesh thus determined is shown in Fig. 2e, and the errors computed are (Fig. 2d) $\epsilon^{*}=5.2 \%$ and $i_{\text {lime }}^{*}=0.43 \%$.

To control the optimality of the mesh, a simple method consists of determining again, neglecting the errors due to the time discretization, a map of optimal sizes for a prescribed accuracy equal to the obtained accuracy $\epsilon^{*}$. If the built mesh is correctly optimized, the procedure must for each element yield a coefficient of modification of size $r_{E}$ close to 1 . In practice, a mesh is correctly optimized if, for the majority of the elements, $0.75 \leqslant r_{E} \leqslant 1.5$. Figure $2 \mathrm{f}$ shows that the mesh $\mathbf{T}^{*}$ is very well optimized.
Secondly, let us consider a frame beam subjected to two loadings (Fig. 3a). The evolution of these loadings is shown in Fig. 3b. The initial mesh used (Fig. 3c) has 387 six-node triangular elements, and the time discretization has 34 time steps. The prescribed accuracy is $\epsilon_{0}=5 \%$. The errors computed are (Fig. 3d) $\epsilon=15.6 \%$ and $i_{\text {ime }}=0.87 \% . i_{\text {ime }}$ may again be neglected and step (2) may be applied. The optimized mesh thus determined is shown in Fig. 3e, and the errors computed are (Fig. 3d) $\epsilon^{*}=5.5 \%$ and $i_{\text {ime }}^{*}=0.79 \%$. The optimality map is shown in Fig. 3f, and the plastic zone size at the end of the loading is shown on the optimized mesh in Fig. 3g.

These examples demonstrate the efficiency of this simple procedure. Yet it is clear that the main drawback of this technique is to neglect the possibilities of optimizing the time discretization.

\subsection{Adaptation of the space-time parameters}

The idea is to use the decomposition $\mathbf{e}^{2}=I_{\text {space }}^{2}+I_{\text {time }}^{2}$ introduced in eqn (26) more completely. Let us recall that evaluating $\mathbf{I}_{\text {time }}$ with $\mathbf{i}_{\text {time }}$ results in the following evaluation for $\mathbf{I}_{\text {space: }}$ :

$$
\mathbf{I}_{\text {space }}^{2}=\sup \left\{\mathbf{e}^{2}-\mathbf{i}_{\text {time }}^{2}, 0\right\} \text {. }
$$

These quantities may be associated to relative quantities by dividing them by $D$, the denominator defined in eqn (14):

$$
\epsilon=\frac{\mathbf{e}}{D} \quad I_{\text {time }}=\frac{\mathbf{I}_{\text {time }}}{D} \quad I_{\text {spacc }}=\frac{I_{\text {space }}}{D} .
$$

Then, for a prescribed global error of $\epsilon_{0}$, the following procedure may be defined:

(1) An initial analysis over the time interval $[0, T]$ is performed on a mesh $T_{e}$ (previously optimized in elasticity) for a time discretization $\Delta$. Then the global error $\epsilon$ and the indicator in time $i_{\text {time }}$ are computed.

(2) A new mesh $\mathrm{T}^{*}$ and a new time discretization $\Delta^{*}$ are determined in order to satisfy

$$
\left\{\begin{array}{l}
\epsilon^{* 2}=\epsilon_{0}^{2} \\
I_{\text {time }}^{2}=\alpha \epsilon_{0}^{2}
\end{array}\right.
$$

where $\alpha$ is a parameter such that $0 \leqslant \alpha \leqslant 1$.

Firstly, the time discretization $\Delta^{*}$ is computed in order that $I_{\text {time }}=\alpha \epsilon_{0}^{2}$. So the length of the time step is uniformly determined by

$$
\Delta t^{*}=\frac{\epsilon_{0}}{I_{\text {time }}} \sqrt{\alpha} \Delta t
$$

Obviously, this modification of the time step is not performed on the first elastic increments.

Secondly, the mesh $T^{*}$ is built $[4,21]$ for a prescribed spatial error $I_{\text {space }}^{2}=(1-\alpha) \epsilon_{0}^{2}$. 

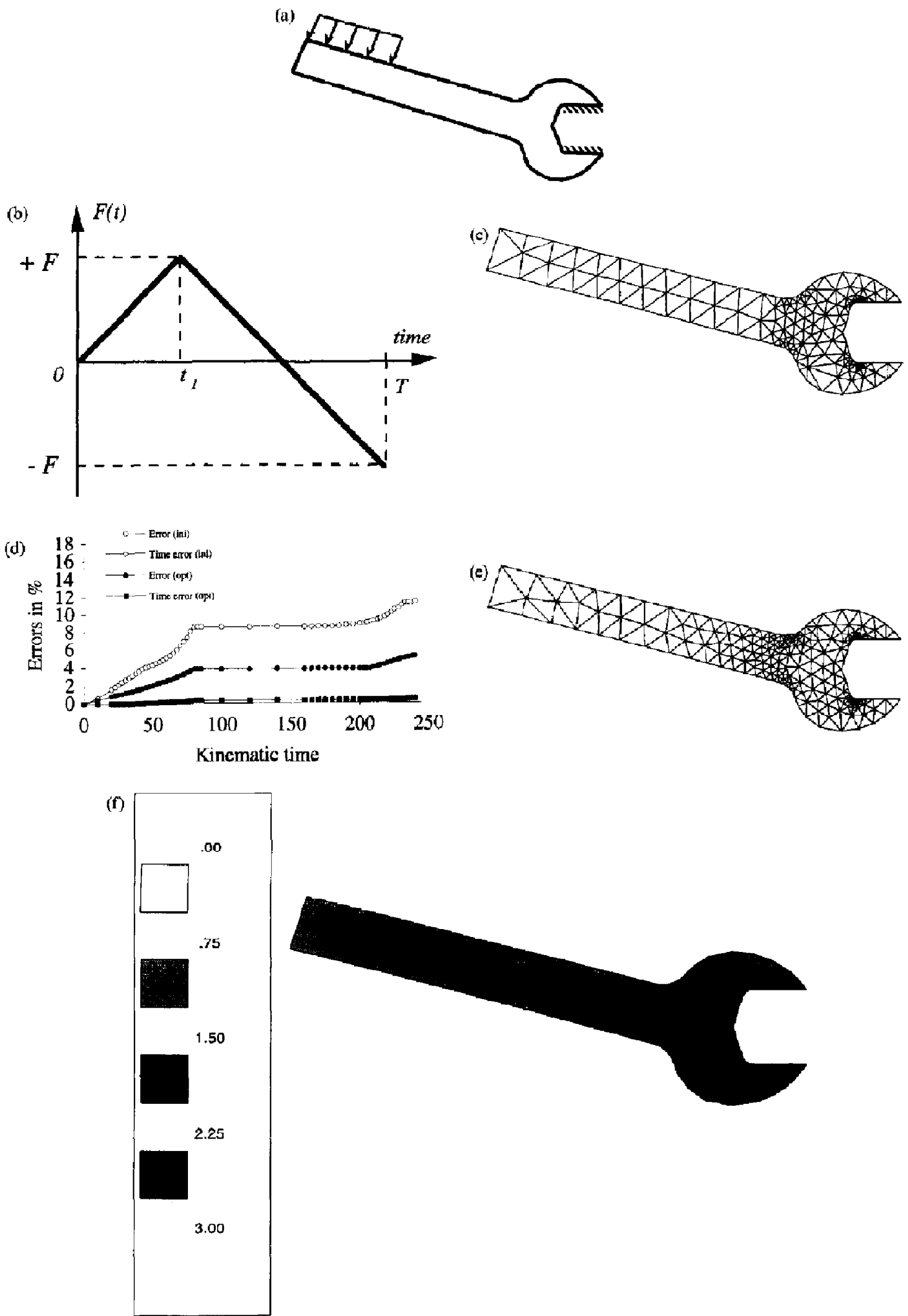

Fig. 2. (a) Mechanical problem; (b) loading: (c) initial mesh-278 six-node trimgular elemenls - 643 nodes; error $11.4 \%$-time error indicutor $0.53 \%$; (d) contributions $\varepsilon_{[0,1]}$ and $t_{\text {time }}, 0$, initial mesh and optimized mesh; (e) optimized mesh-423 six-node triangular elements - 950 nodes, error $5.2 \%$-time error indicator $0.43 \%$, prescribed error $5.0 \%$; (f) optimality map. 

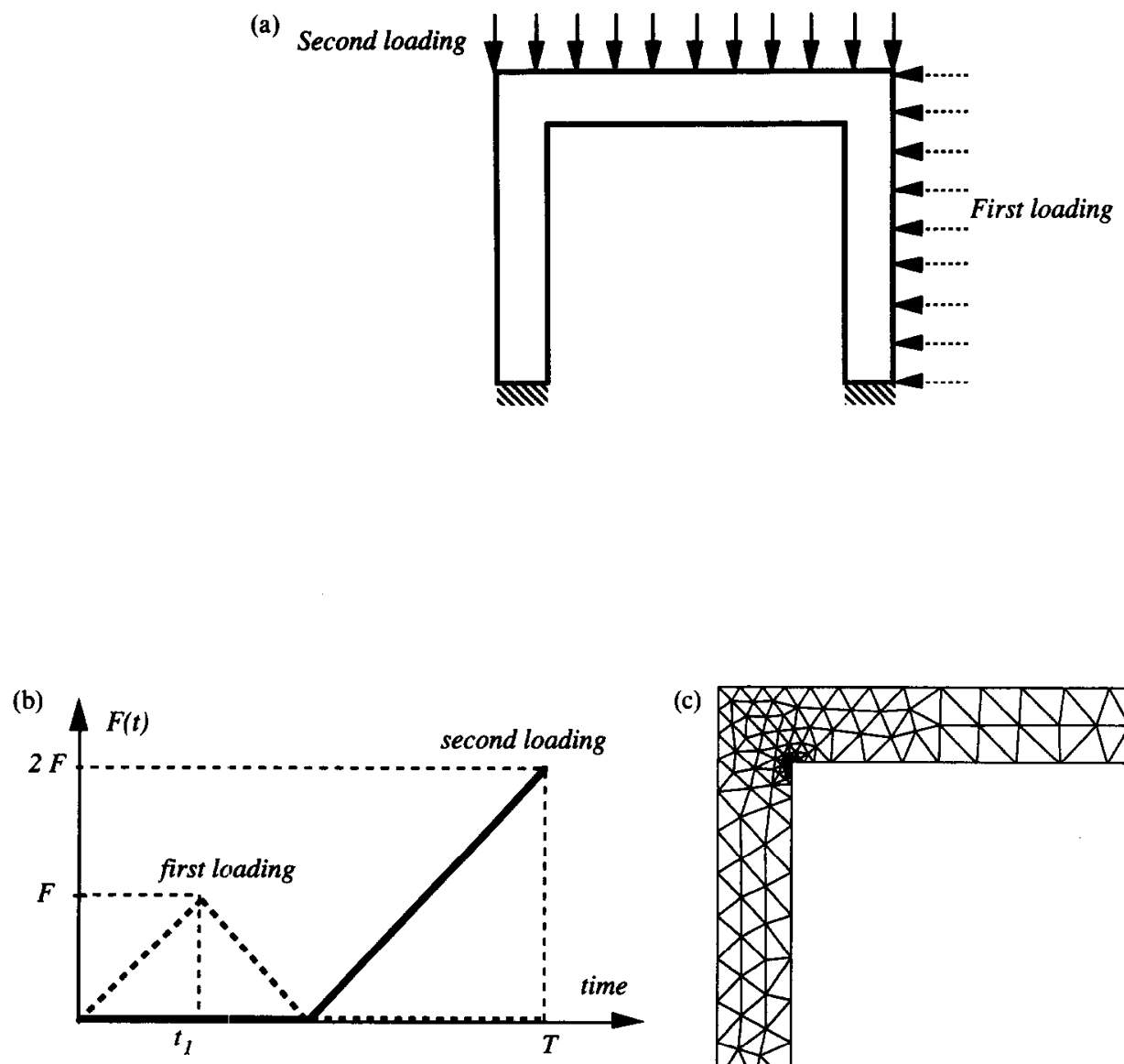

(c)
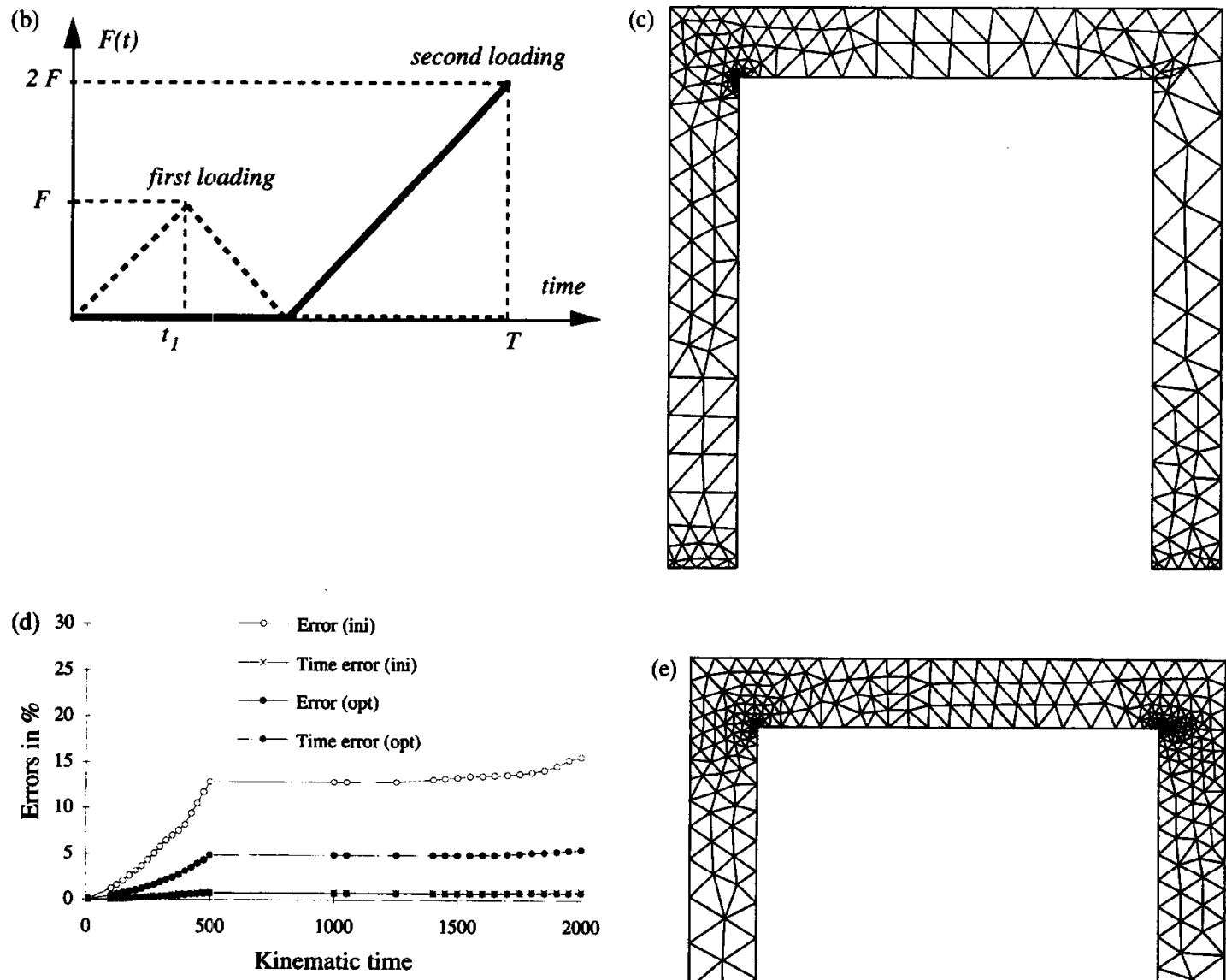

(e)

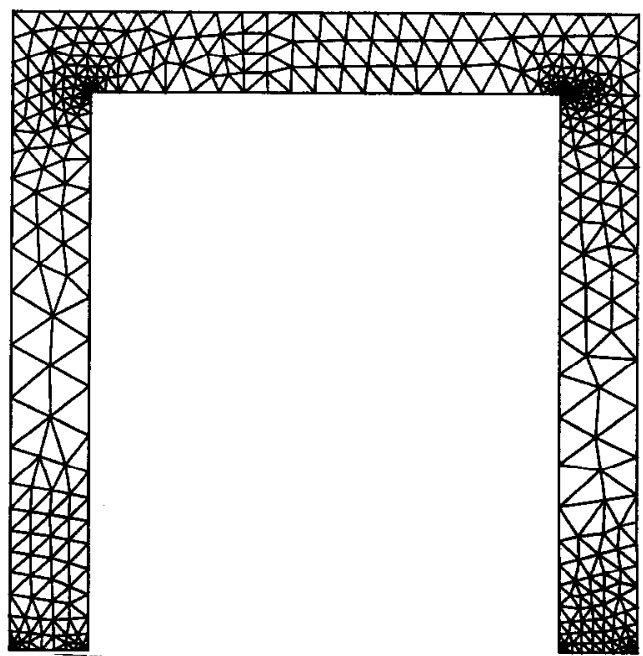

Fig. 3. (a) Mechanical problem; (b) evolution of the loadings as a function of time; (c) initial mesh-387 six-node triangular elements- -890 nodes, error $15.6 \%$-time error indicator $0.87 \%$; (d) contributions $\epsilon_{f o, l}$ and $i_{\text {ime. } \mid 0.1)}$, initial mesh and optimized mesh; (e) optimized mesh-824 six-node triangular elements -1827 nodes, error $5.5 \%$ - time error indicator $0.79 \%$, prescribed error $5.0 \%$; (f) optimality map; (g) size of the plastic zone: initial threshold $100 \mathrm{MPa}$. (Continued overleaf.) 
(f)

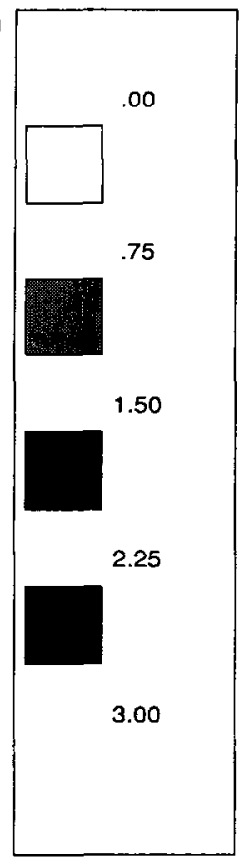

(g)

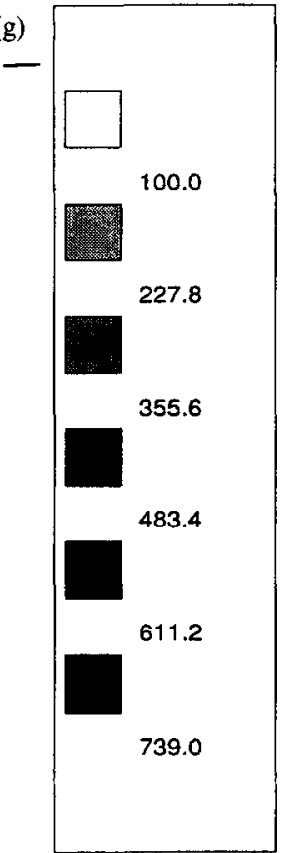

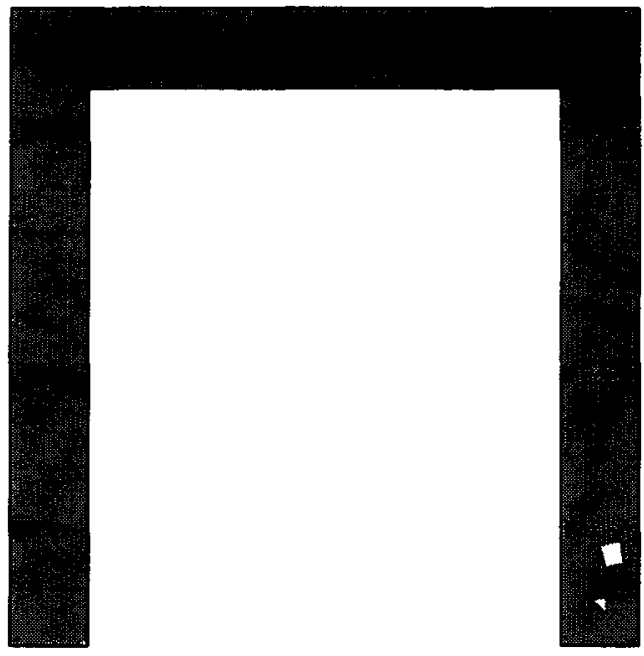

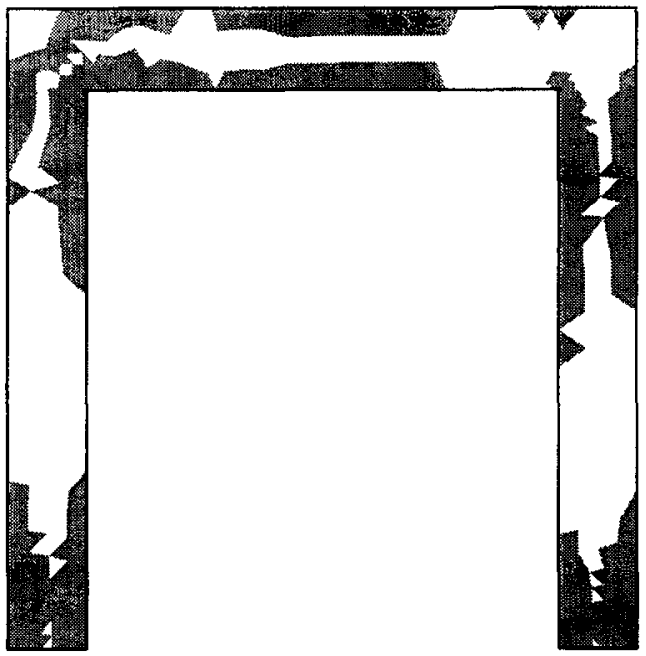

Fig. 3-Continued.
Remarks: (1) a limit $\Delta t_{\max }$ is necessarily fixed to the length of $\Delta t^{*}$ in order to correctly represent the history of the loading on each time step. If this maximum length is reached, then $\alpha$ must be chosen as

$$
\alpha \leqslant\left[\frac{\Delta t_{\max }}{\Delta t} \frac{I_{\text {time }}}{\epsilon_{0}}\right]^{2} .
$$

(2) $\alpha$ is a parameter which can be adjusted in order to reduce the cost of the analysis to the maximum extent possible.

Let us examine the example shown in Fig. 4a. The loading is monotonous, and the initial analysis is conducted with a 129 six-node element mesh (Fig. 4b) and a sharp time discretization (30 time-steps). The prescribed error is $2 \%$. In this example, we choose 
$\alpha=0.5$, which means that we fix beforehand $I_{\text {space }}=\mathbf{I}_{\text {time }}$. The initial analysis leads to $\epsilon=5.4 \%$ and $i_{\text {time }}=0.67 \%$. The simultaneous adaptivity procedure leads to the use of a 15-increment time discretization with a 324-element optimized mesh, as shown in Fig. 4c. The errors computed are then $\epsilon^{*}=2.5 \%$ and $i_{\text {time }}^{*}=1.2 \%$. Figure $4 \mathrm{~d}$ shows the evolution of the contributions $\epsilon_{[0, i]}$ and $i_{\text {time, }[0, i]}$ on the initial and optimized mesh. The plastic zone at the end of

(a)
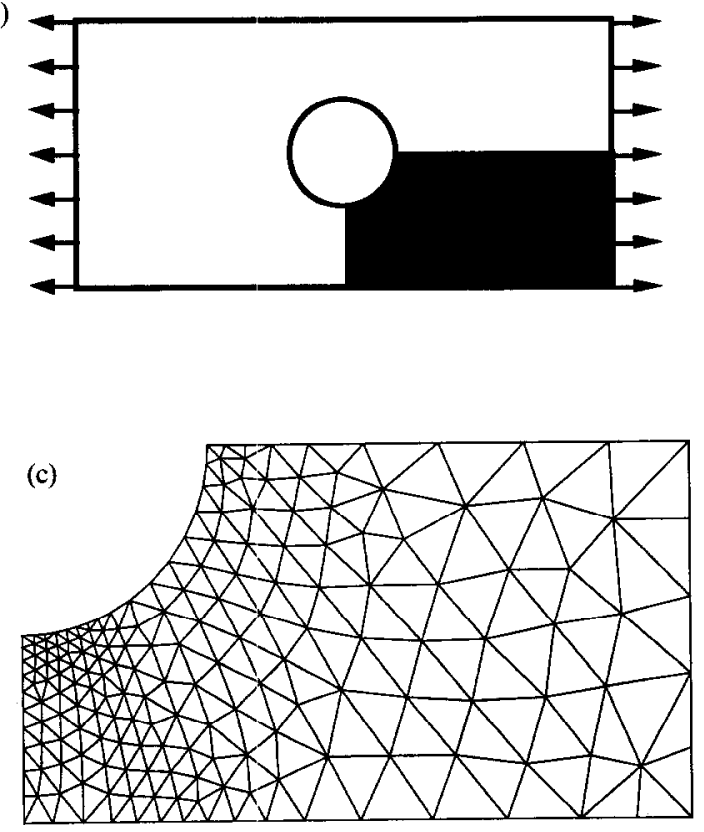

(d)
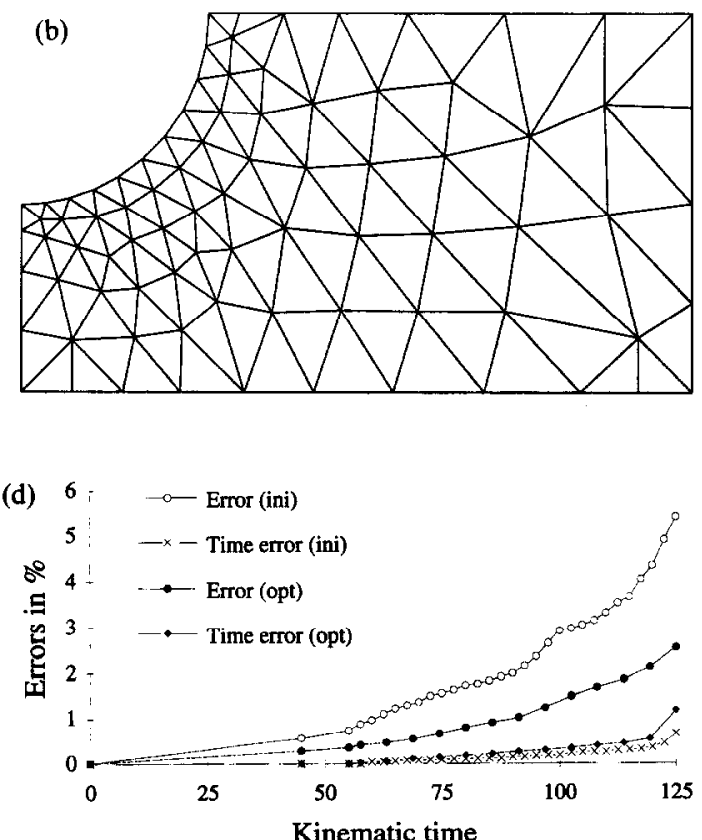

(e)

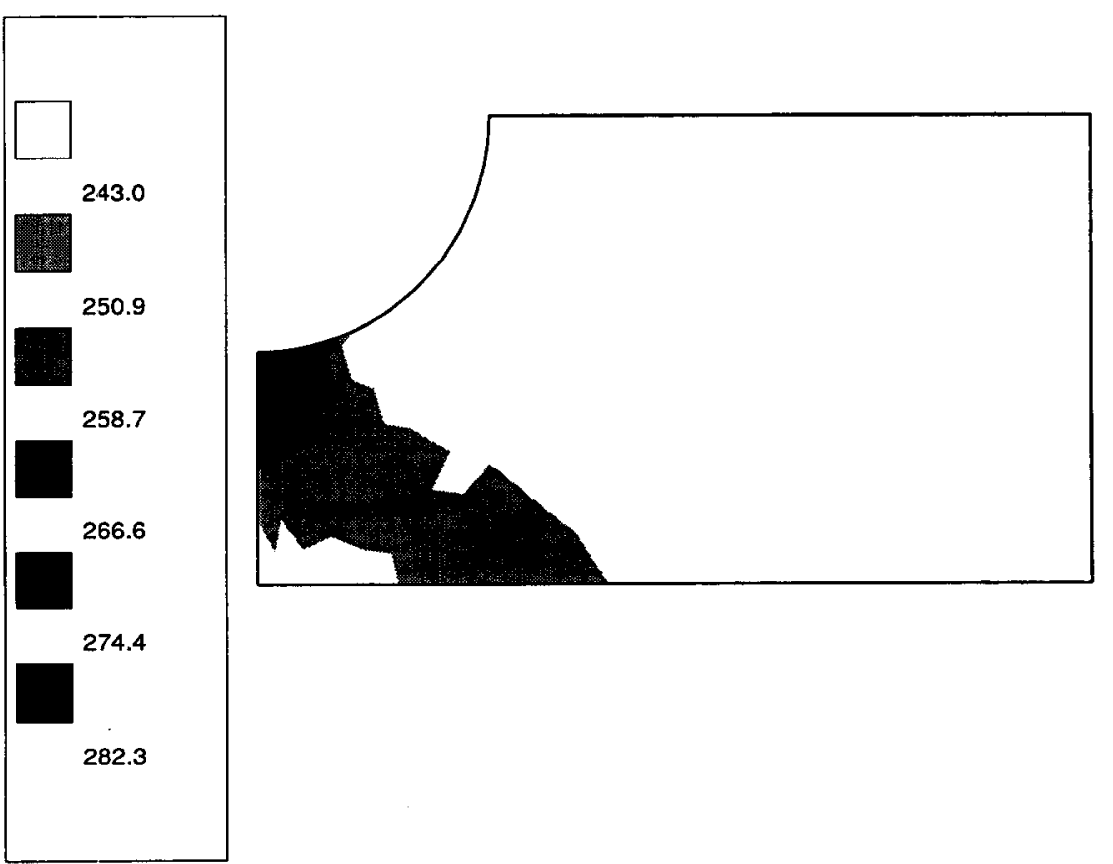

Fig. 4. (a) Mechanical problem; (b) initial mesh-129 six-node triangular elements - 294 nodes, error $5.4 \%$-time error indicator $0.67 \%$; (c) optimized mesh-324 six-node triangular elements -705 nodes, error $2.5 \%$-time error indicator $1.2 \%$, prescribed error $2.0 \%$; (d) contributions $\epsilon_{[0,1]}$ and $i_{\text {time. }[0 .,]}$, initial mesh: 30 increments, optimized mesh: 15 increments; (e) size of the plastic zone: initial threshold $243 \mathrm{MPa}$. 
$5.4 \%$, and $243 \mathrm{~s}$ for the final computation which leads to a precision of $2.5 \%$. Hence, in this example, we obtain a better approximation with a lower computation time.

\section{CONCLUSION}

An error measure which allows one to take into account all the errors due to the discretization (errors due to the spatial discretization, errors due to the incremental method) has been implemented in elasto-plasticity.

A time error indicator based on the concept of error in constitutive relation has been proposed. This indicator permits the separation of the contribution to the error due to the spatial discretization from the contribution due to the incremental method. It has been used to develop two simple procedures of adaptivity of the time-space parameters for a finite element non-linear computation.

The first one imposes the use of sufficiently fine time-discretization in order to neglect the associated contribution when compared to the error due to the spatial discretization. In this case, the adaptivity of the computation is confined to the adaptivity of the mesh. The second one is a first example of a procedure that permits the simultaneous adaptation of the mesh and of the length of the time increments. The examples presented prove the efficiency of the two procedures.

As an initial step these procedures have been implemented for space discretizations that use threeor six-node elements in two-dimensional or axisymmetric analysis, and for Prandtl-Reuss's constitutive relation in elasto-plasticity.

\section{REFERENCES}

1. P. Ladevèze and D. Leguillon, Error estimate in the finite element method and applications. SIAM J. numer. Anal. 20, 485-509 (1983).

2. D. W. Kelly, J. P. Gago, O. C. Zienkiewicz and I. Babuska, $A$ posteriori error analysis and adaptive processes in the finite element method: part I-error analysis. Int. J. numer. Meth. Engng 19, 1593-1619 (1983).

3. O. C. Zienkiewicz and J. Z. Zhu, A simple error estimator and adaptive procedure for practical engineering analysis. Int. J. numer. Meth. Engng 24, 337-357 (1987).

4. G. Coffignal and P. Ladevèze, Error computation and optimal mesh in elasticity and elastoplasticity. In: Proc. SMIRT 7, Chicago, vol. L, pp. 177-182. NorthHolland, Amsterdam (1983).

5. P. Ladevèze, G. Coffignal and J. P. Pelle, Accuracy of elastoplastic and dynamic analysis. In: Accuracy
Estimates and Adaptivity for Finite Elements (Edited by 1. Babuska, O. C. Zienkiewicz, J. P. Gago and A. E. Oliveira), chap. 10, pp. 181-203. Wiley, New York (1986).

6. W. K. Liu, T. Belytschko and H. Chang, An arbitrary Lagrangian-Eulerian finite element method for pathdependent materials. Comput. Meth. appl. Mech. Engng 58, 227-245 (1986).

7. J. T. Oden, T. Strouboulis and P. Devloo, Adaptive finite element methods for the analysis of inviscid compressible flow: part I. Fast refinement/unrefinement and moving mesh methods for unstructured meshes. Comput. Meth. appl. Mech. Engng 59, 327-362 (1986).

8. J. M. Bass and J. T. Oden, Adaptive finite methods for a class of evolution problems in viscoplasticity. Int. J. Engng Sci. 6, 623-653 (1987).

9. O. C. Zienkiewicz, Y. C. Liu and G. C. Huang, Error estimation and adaptivity in flow formulation for forming problems. Int. J. numer. Meth. Engng 25, 23-42 (1988).

10. C. Johnson and P. Hansbo, Adaptive finite element methods for small strain elasto-plasticity. In: IUTAM 91, Proc. Conf. on Finite Inelastic DeformationsTheory and Applications, University of Hannover, Hannover, pp. 273-288 (1991).

11. M. Ortiz and J. J. Quigley, Adaptive mesh refinement in strain localization problems. Comput. Meth. appl. Mech. Engng 90, 781-804 (1991).

12. D. Aubry and B. Tie, $A$ posteriori error analysis and $h$ adaptive refinement for the FEM in non-linear structural computations. In: Proc. European Conf. on New Advances in Computational Structural Mechanics, Giens, pp. 1-8 (1991).

13. W. Rust and E. Stein, Two-dimensional finite element mesh adaptations in structural mechanic, including shell analysis and non-linear calculations. In: New Advances in Computational Structural Mechanics (Edited by P. Ladevèze and O. C. Zienkiewicz), pp. 219-232. Elsevier, New York (1991).

14. D. R. J. Owen, D. Peric and J. Yu, On error estimate and adaptivity in elasto-plastic solids: applications to the numerical simulation of strain localization in classical and cosserat continua. Int. J. numer. Meth. Engng 37, 1351-1379 (1994).

15. L. Gallimard, P. Ladevèze and J. P. Pelle, Error estimation and adaptivity in elastoplasticity Int. $J$. numer. Meth. Engng 39, 189-217 (1996).

16. P. Ladevèze, Sur une famille d'algorithmes en mécanique des structures. C. R. Acad. Sci. Paris II 300, 41-44 (1985).

17. D. C. Drucker, On the postulate of stability of materials in the mechanics of continua. $J$. Méc. 3, 235-245 (1964).

18. D. R. J. Owen and E. Hinton, Finite Elements in Plasticity, 2nd edn. Pineridge, Swansea (1986).

19. K. J. Bathe, Finite Element Procedures in Engineering Analysis. Prentice-Hall, Englewood Cliffs, NJ (1982).

20. L. Gallimard and J. P. Pelle, ERPLAS: Un logiciel pour le contrôle et l'adaptativité des calculs en élastoplasticité. In: StruCome 93, Proc. StruCome 93, Paris, pp. 265-276 (1993)

21. P. Ladevèze, J. P. Pelle and P. Rougeot, Error estimation and mesh optimization for classical finite elements. Engng Comput. 8, 69-80 (1991). 